\title{
REPRESENTATIONS OF ANISOTROPIC UNITARY GROUPS
}

\author{
DONALD G. JAMES
}

\begin{abstract}
Let $S U(f)$ be the special unitary group of an anisotropic hermitian form $f$ over a field $k$. Assume $f$ represents only one norm class in $k$. The representations $\alpha: S U(f) \rightarrow S L(n, R)$ are characterized when $R$ is a commutative ring with 2 not a zero divisor and $n=\operatorname{dim} f \geq 3$ with $n \neq 4,6$. In particular, a partial classification of the normal subgroups of $S U(f)$ is given when $k$ is the function field $\mathbf{C}(X)$.
\end{abstract}

1. Introduction. We study representations $\alpha: U(f) \rightarrow G L(n, R)$ where $U(f)$ is the unitary or orthogonal group of an anisotropic form $f$ over a field $k$ with $\operatorname{dim} f=n \geq 3$ and $R$ is a commutative ring with 2 not a zero divisor. Representations of the special unitary group $S U(f)$ will also be considered and, in particular, a partial classification of the normal subgroups of $S U(f)$ is obtained. However, the method only applies to a restricted class of fields $k$, including all real closed fields. The results are first established for $R$ a local ring using a generalization of the fundamental theorem of projective geometry to construct a generalized place $\varphi: k \rightarrow R \cup \infty$ with the inverse image $A=\varphi^{-1}(R)$ a valuation ring of $k$. The kernel of the homomorphism $\varphi: A \rightarrow R$ is now an ideal $\mathfrak{a}$ of $A$ which need not be maximal. The kernel of the homomorphism $\alpha$ is a twisted congruence subgroup $U(\mathfrak{a}, \chi)$ of $U(f)$ defined with respect to $\mathfrak{a}$ and a character $\chi: U(f) \rightarrow \mathfrak{u}(R)$, where $\mathfrak{u}(R)$ denotes the units of $R$. For general commutative rings $R$, the kernel of $\alpha$ is the intersection of the local kernels obtained after $\alpha$ is extended by localizing at the maximal ideals of $R$. These results generalize earlier work of Weisfeiler [16] and James $[\mathbf{9}, \mathbf{1 0}]$ where $R$ is a field. Earlier, Borel and Tits $[\mathbf{3}]$ had studied abstract homomorphisms of isotropic algebraic groups and Tits [15] had considered representations of Lie groups. See [8] for a general survey of this area.

Let $k$ be a field of characteristic not two with involution ${ }^{*}, V$ a $k$-space of finite dimension $n \geq 3$ and $f: V \times V \rightarrow k$ an anisotropic hermitian form. Thus $f(x, x)=0$ implies $x=0$. Let $U(f)$ be the unitary group of $f$ and $I(f)$ the subgroup of $U(f)$ generated by involutions. We allow the involution ${ }^{*}$ on $k$ to be trivial, in which case $f$ becomes a quadratic form and $U(f)=I(f)$ is an orthogonal group. The symmetry $\Psi(x): y \mapsto y-2 f(x, y) f(x, x)^{-1} x$ is an involution in $U(f)$ for each nonzero $x$ in $V$. Henceforth we only consider forms $f$ for which all symmetries in $U(f)$ are conjugate under $S I(f)=I(f) \cap S U(f)$. By Witt's Theorem this means that $f$ can only represent one norm class (or square class when ${ }^{*}$ is trivial). Then necessarily $f$ can be diagonalized as a sum of norms (or squares) and, since $f$ is anisotropic, $k$ will have characteristic zero. If $f$ is a quadratic form then $k$ must be

Received by the editors March 3, 1987.

1980 Mathematics Subject Classification (1985 Revision). Primary 20G05, 51A10; Secondary $11 \mathrm{E} 57$.

This research was partially suported by the National Science Foundation. 
pythagorean and formally real (see Lam [12]); this includes all real closed fields, as well as fields of Laurent series in several variables over $\mathbf{R}$ or any other real closed field. When $f$ is hermitian there are more possibilities. Now we can choose $k=K(a)$, with $K$ formally real pythagorean and $a \notin K$ with $a^{2} \in K$, or $K$ any formally real field with pythagoras number two and $a^{2}=-1$. In particular, our results now apply to the function field $\mathbf{C}(X)$ and this case will be considered in greater detail. Some of our results will also hold when $f$ is an hermitian form over a quaternion division algebra of special type (see [10, p. 348]).

It is shown in $[\mathbf{1 0}$, Proposition 3.1] that $U(f)$ is generated by $I(f)$ and any one-dimensional subgroup $U(1)$ of $U(f)$. Since $S I(f)$ is generated by products of two symmetries $\Psi(x) \Psi(y)$ where $\Psi(x)$ and $\Psi(y)$ are conjugate, it follows that $S I(f)$ is contained in the commutator subgroup $[U(f), U(f)]$. If $U(1)$ is the onedimensional subgroup on the subspace $k x$ of $V$ then $\Psi(x) \in U(1)$. Hence the map det: $U(f) \rightarrow k^{*}$ has kernel $S I(f)$. Thus $S I(f)=S U(f)$ is the commutator subgroup of $U(f)$ and

$$
1 \rightarrow S U(f) \rightarrow U(f) \stackrel{\text { det }}{\longrightarrow} N_{1}(k) \rightarrow 1
$$

is an exact sequence, where $N_{1}(k)=\left\{a \in k \mid a^{*} a=1\right\}$ is the norm one subgroup of $k^{*}$. It is also easily seen that $S U(f)$ is perfect.

Our main result is the following.

THEOREM 1. Let $f$ be an anisotropic hermitian form which represents only one norm class and let $G$ be a subgroup of $U(f)$ containing $S U(f)$. Let $\alpha: G \rightarrow G L(M)$ be a representation of $G$ where $M$ is a free module over a commutative local ring $R$ with 2 a unit and $\alpha S U(f) \neq 1$. Assume $\operatorname{dim} f=\operatorname{rank} M=n \geq 3$ and $n \neq 4,6$. Then there exist

(i) a generalized place $\varphi: k \rightarrow R \cup \infty$ with $A=\varphi^{-1}(R)=A^{*}$ a valuation ring and $\varphi: A \rightarrow R$ a homomorphism with kernel $\mathfrak{a}$,

(ii) a free maximal A-lattice $N$ in $V$ of rank $n$ with $U(N)=U(f)$,

(iii) a $\varphi$-semilinear map $\beta: N \rightarrow M$ with kernel $\mathfrak{a} N$,

(iv) a character $\chi: G \rightarrow \mathfrak{u}(R)$,

(v) a twisted congruence subgroup $G(\mathfrak{a}, \chi)$ of $G$, such that

$$
1 \rightarrow G(\mathfrak{a}, \chi) \rightarrow G \stackrel{\alpha}{\rightarrow} G L(M)
$$

is an exact sequence. Moreover, for each $\sigma \in G$ and $x \in N,(\alpha \sigma) \beta(x)=\chi(\sigma) \beta \sigma(x)$. In particular, when $G=S U(f)$, the diagram

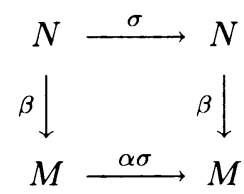

is commutative.

The theorem is false when $n=4$ and $G=S U(f)$, see Artin [1, Theorem 5.23] since there are now nontrivial homomorphisms $\alpha: S O(f) \rightarrow S L(3, k)$ with kernel not a congruence subgroup. Probably the theorem is true when $n=6$, but there are some technical difficulties at one point in our method of proof. However, if we 
modify the assumptions on $G$ to $I(f) \subseteq G$ and card $\alpha I(f)>2$, then the theorem remains true for both $n=4$ and 6 .

Theorem 1 can be used, with the help of a localization argument, to give the following partial classification of the normal subgroups of $S U(f)$ in terms of congruence subgroups.

THEOREM 2. Let $f$ be an anisotropic hermitian form which represents only one norm class. Assume $\operatorname{dim} f=n \geq 3$ and $n \neq 4,6$. Let $H$ be a nontrivial normal subgroup of $S U(f)$ such that $S U(f) / H$ is contained in $G L(n, R)$ for some commutative ring $R$ with 2 not a zero divisor. Then $H$ is the intersection of a family of congruence subgroups of the type $S U(\mathfrak{a}, 1)$ in Theorem 1.

Special cases of Theorem 2 are known for orthogonal groups without the restriction $S O(f) / H \subseteq G L(n, R)$. When $k=\mathbf{R}$ the projective group $P S O(f)$ is simple, $n \neq 4$ (see [1]). Pollak [14] considered the field $k=\mathbf{R}((X))$ of formal Laurent series and Chang $[\mathbf{5}]$ generalized this to several variables. Archimedean ordered pythagorean fields are considered by Bröcker [4]. Probably their results can also be modified to unitary groups.

For specific fields $k$ the results in Theorems 1 and 2 can be strengthened. In particular, if $\mathbf{R}$ is contained in the fixed field of $k$, then the valuation on $\mathbf{R}$ induced by the restricted place is trivial (Proposition 8).

We will consider unitary groups over the rational function field $k=\mathbf{C}(X)$, with involution induced by $i^{*}=-i$, in detail. For each complex number $c$ the identity map $\varphi_{c}: \mathbf{C} \rightarrow \mathbf{C}$ extends to a unique place $\varphi_{c}: k \rightarrow \mathbf{C} \cup \infty$ by setting $\varphi_{c}(X)=c$. The associated valuation ring $A_{c}$ is the set of rational functions $r(X)=g(X) / h(X)$ over $\mathbf{C}$ with $g(X), h(X)$ relatively prime polynomials in $\mathbf{C}[X]$ and $h(c) \neq 0$. Fix an integer $m \geq 1$ and let $R(c, m)$ denote the local ring $\mathbf{C}[X] /(X-c)^{m}$. Then a generalized place $\varphi_{c, m}: k \rightarrow R(c, m) \cup \infty$ can be obtained as follows. If $r(X) \notin A_{c}$ set $\varphi_{c, m}(r(X))=\infty$. Any $r(X) \in A_{c}$ can be expanded as a unique formal power series $r(X)=\sum_{j=0}^{\infty} a_{j}(X-c)^{j}$ with $a_{j} \in \mathbf{C}$. Set $\varphi_{c, m}(r(X))=\sum_{j=0}^{m-1} a_{j}(X-c)^{j}$, viewed as an element in $R(c, m)$. The kernel of $\varphi_{c, m}: A_{c} \rightarrow R(c, m)$ is the ideal $(X-c)^{m} A_{c}$. Another place $\varphi_{\infty}$ on the field $\mathbf{C}(X)$ can be obtained by setting $\varphi_{\infty}(X)=\infty$; the associated valuation ring $A_{\infty}$ consists of all rational functions $r(X)$ with $\operatorname{deg}(r(X)) \leq 0$. For each integer $m \geq 1$ there corresponds a generalized place $\varphi_{\infty, m}$. The kernel of the restriction of $\varphi_{\infty, m}$ to $A_{\infty}$ consists of all rational functions $r(X)$ with $\operatorname{deg}(r(X)) \leq-m$.

Now let $A$ be the intersection of a finite number of the valuation rings of $\mathbf{C}(X)$ just considered with $\varphi(X)$ real or infinite. Then $A$ is a semilocal ring which can be described as follows. Choose a finite number of real numbers $c_{1}, \ldots, c_{l}$ (possibly none) and also possibly choose $\infty$. Then $A$ consists of all rational functions $r(X)=$ $g(X) / h(X)$ with $h\left(c_{i}\right) \neq 0,1 \leq i \leq l$, and also $\operatorname{deg}(r(X)) \leq 0$ if $\infty$ is chosen. Let $V$ be a vector space over $\mathbf{C}(X)$ with basis $u_{1}, \ldots, u_{n}, n \geq 3$, and let $f$ be the anisotropic hermitian form defined by $f\left(u_{i}, u_{j}\right)=\delta_{i j}, 1 \leq i, j \leq n$. Then $N=A u_{1} \perp \cdots \perp A u_{n}$ is a maximal lattice in $V$ consisting of all $x \in V$ with $f(x, x) \in A$ (if any $c_{i}$ is nonreal, $N$ is not maximal). Hence $U(N)=U(f)$ where $U(N)$ consists of all $\sigma \in U(f)$ with $\sigma(N)=N$. Let $\mathfrak{a}$ be an ideal of the principal ideal ring $A$ and define $S U(\mathfrak{a})$ as the congruence subgroup of $S U(f)$ consisting of 
all $\sigma \in S U(f)$ for which $\left(\sigma-1_{V}\right)(N) \subseteq \mathfrak{a} N$. Then we have an exact sequence

$$
1 \rightarrow S U(\mathfrak{a}) \rightarrow S U(f) \rightarrow S L(n, R)
$$

where $R$ is the commutative $\operatorname{ring} A / \mathfrak{a}$. The ideal $\mathfrak{a}$ is generated by a rational function $r(X)$ of the form

$$
r(X)=\left(X-c_{1}\right)^{m_{1}} \cdots\left(X-c_{l}\right)^{m_{l}} /(X-c)^{m+m_{1}+\cdots+m_{l}}
$$

with $c_{1}, \ldots, c_{l}, c$ all real and distinct; this gives the connection with the generalized places.

THEOREM 3. Let $H$ be a nontrivial normal subgroup of the special unitary group $S U(f)$ over the field $\mathbf{C}(X)$. Assume $n=\operatorname{dim} f \geq 3, n \neq 4,6$, and $S U(f) / H \subseteq$ $G L(n, R)$ for some commutative ring $R$ with 2 not a zero divisor. Then $H$ is a congruence subgroup $S U(\mathfrak{a})$.

The above three theorems are a further contribution to the solutions of very general questions asked by Weisfeiler [11, XVII and 17, §7], although we have gone beyond his original framework in allowing the image group to be defined over a ring.

2. Collineations and projective geometry. Let $R$ be a local ring with maximal ideal $\mathfrak{m}=\mathfrak{m}(R)$. In this section it is not necessary to assume that $R$ is commutative or that 2 is a unit. Let $M$ be a free $R$-module of finite rank $n \geq 3$. A point of $M$ is a rank one direct summand of $M$ and a line is a rank two direct summand of $M$. The projective space $\mathbf{P} M$ of $M$ is the set of all points of $M$. A projective frame of $\mathbf{P} M$ is a set of $n$ points $P_{1}, \ldots, P_{n}$ such that $M=$ $P_{1}+P_{2}+\cdots+P_{n}$. A special case of the above situation is a vector space $V$ over a (skew) field $k$ with associated projective space $\mathbf{P} V$.

DEFINITION. A collineation (or projective homomorphism) is a map $\pi: \mathbf{P} V \rightarrow$ $\mathbf{P} M$ sending points of $\mathbf{P} V$ to points of $\mathbf{P} M$ such that

(i) there exists a projective frame of $\mathbf{P} V$ which is carried by $\pi$ to a projective frame of $\mathbf{P} M$ (and hence $\operatorname{dim} V=\operatorname{rank} M$ ),

(ii) if the images of the points $P_{1}$ and $P_{2}$ of $\mathrm{P} V$ generate a line $\pi P_{1}+\pi P_{2}$ in $\mathbf{P} M$, then any point $P$ on the line $P_{1}+P_{2}$ has image $\pi P$ on the line $\pi P_{1}+\pi P_{2}$.

If, moreover, in (ii) above, there always exists a point $P_{3}$ on the line $P_{1}+P_{2}$ such that $\pi P_{1}+\pi P_{2}=\pi P_{1}+\pi P_{3}=\pi P_{2}+\pi P_{3}$, then the collineation $\pi$ is called thick.

If $P_{1}$ and $P_{2}$ are distinct points in $\mathrm{P} V$ then $P_{1}+P_{2}$ is always a line. However, this is false in $\mathbf{P} M$ since the span of the points need not be a direct summand of $M$. This is the reason for the more careful phrasing in the definition of a collineation.

Fundamental Theorem. Let $\pi: \mathbf{P} V \rightarrow \mathbf{P} M$ be a thick collineation. Then there exist

(i) a generalized place $\varphi: k \rightarrow R \cup \infty$ with $A=\varphi^{-1}(R)$ a valuation ring of $k$ and the restriction $\varphi: A \rightarrow R$ a ring homomorphism,

(ii) a free $A$-module $N$ in $V$ with $\operatorname{rank} N=\operatorname{dim} V$,

(iii) a $\varphi$-semilinear map $\beta: N \rightarrow M$, such that $\beta$ induces $\pi$, namely, $\pi P=R \beta(P \cap N)$ for $P \in \mathbf{P} V$.

PROOF. Choose a basis $u_{1}, \ldots, u_{n}$ in $V$ such that $\pi\left(k u_{i}\right)=R v_{i}, 1 \leq i \leq n$, is a projective frame for $\mathbf{P} M$. Thus $M=R v_{1}+\cdots+k v_{n}$. Fix $j$ with $2 \leq j \leq n$. 
Since $R v_{1}$ and $R v_{j}$ span a line, it follows from the definition of a thick collineation that there exists a point $P_{3}=k\left(u_{1}+a u_{j}\right)$ in $\mathbf{P} V, a \neq 0$, with image $R\left(b v_{1}+c v_{j}\right)$ satisfying

$$
R v_{1}+R v_{j}=R v_{1}+R\left(b v_{1}+c v_{j}\right)=R v_{j}+R\left(b v_{1}+c v_{j}\right)
$$

Hence $b$ is a unit in $R$ and we may assume $b=1$. Also $c$ is a unit in $R$. Replacing $u_{j}$ by $a^{-1} u_{j}$ and $v_{j}$ by $c^{-1} v_{j}$ we can normalize our choice of basis $v_{1}, \ldots, v_{n}$ for $M$ such that

$$
\pi\left(k\left(u_{1}+u_{j}\right)\right)=R\left(v_{1}+v_{j}\right), \quad 2 \leq j \leq n .
$$

For each $a \in k$ the point $k\left(u_{1}+a u_{j}\right), j \neq 1$, lies on the line $k u_{1}+k u_{j}$ and hence its image is either $R\left(v_{1}+b v_{j}\right)$ for some $b \in R$, or $R\left(p v_{1}+v_{j}\right)$ for some $p \in \mathfrak{m}$. In the first case set $\varphi_{j}(a)=b$ and in the second case set $\varphi_{j}(a)=\infty$. Then for each $j \neq 1$ we have defined a map $\varphi_{j}: k \rightarrow R \cup \infty$ with $\varphi_{j}(0)=0$ and $\varphi_{j}(1)=1$. Let $A_{j}=\varphi_{j}^{-1}(R)$. We now prove that $\varphi_{j}=\varphi$ and $A_{j}=A$ are independent of $j$, that $A$ is a valuation ring of $k$ and $\varphi: A \rightarrow R$ is a ring homomorphism.

Fix $1<i \neq j \leq n$ and let $a \in A_{i}$ and $b \in A_{j}$. Then by standard arguments it follows that the images of the points $k\left(u_{1}+a u_{i}+b u_{j}\right)$ and $k\left(a u_{i}+u_{j}\right)$ are respectively, $R\left(v_{1}+\varphi_{i}(a) v_{i}+\varphi_{j}(b) v_{j}\right)$ and $R\left(\varphi_{i}(a) v_{i}+v_{j}\right)$. Hence, for nonzero $a, a^{\prime}$ in $A_{i}$, the image of the point $k\left(u_{1}+\left(a+a^{\prime}\right) u_{i}+u_{j}\right)$ lies on both of the lines $R\left(v_{1}+\varphi_{i}(a) v_{i}\right)+R\left(\varphi_{i}\left(a^{\prime}\right) v_{i}+v_{j}\right)$ and $R\left(v_{1}+\varphi_{i}(a) v_{i}+v_{j}\right)+R v_{i}$. If $\varphi_{i}\left(a+a^{\prime}\right)=\infty$ then this image also lies on the line $R\left(p v_{1}+v_{i}\right)+R v_{j}$ where $p \in \mathfrak{m}$, which leads to a contradiction. Thus $\varphi_{i}\left(a+a^{\prime}\right) \neq \infty$ and $A_{i}$ is closed under addition. It now easily follows that $\varphi_{i}\left(a+a^{\prime}\right)=\varphi_{i}(a)+\varphi_{i}\left(a^{\prime}\right)$.

Again take nonzero $a \in A_{j}, b \in A_{i}$. Since the images of $k u_{1}$ and $k\left(b u_{i}+u_{j}\right)$ span a line in $\mathbf{P} M$, as do the images of $k\left(u_{1}+a u_{j}\right)$ and $k u_{i}$, it follows that the image of $k\left(u_{1}+a b u_{i}+a u_{j}\right)$ is $R\left(v_{1}+\varphi_{j}(a) \varphi_{i}(b) v_{i}+\varphi_{j}(a) v_{j}\right)$. Hence the image of $k\left(u_{1}+a b u_{i}\right)$ cannot be $R\left(p v_{1}+v_{i}\right)$ and so necessarily $a b \in A_{i}$. Letting $b=1$ it follows that $A_{j} \subseteq A_{i}$ and hence $A_{i}=A_{j}=A$ is closed under multiplication. Moreover, $\varphi_{i}(a b)=\varphi_{j}(a) \varphi_{i}(b)$ so that $\varphi_{i}=\varphi_{j}=\varphi$ and $\varphi(a b)=\varphi(a) \varphi(b)$. Note we still have not shown that $A$ is a ring, namely $-1 \in A$.

Next consider $a \notin A$. Then $\pi\left(k\left(u_{1}+a u_{2}\right)\right)=R\left(p v_{1}+v_{2}\right)$ where $p \in \mathfrak{m}$. Repeat all the previous arguments with $u_{1}$ and $u_{2}$ interchanged; there is no need to change the normalization of the basis, however, since it is already established that

$$
\pi\left(k\left(u_{2}+u_{i}\right)\right)=R\left(v_{2}+v_{i}\right) \text { for } i \neq 2 .
$$

We obtain a new set $B \subseteq k$, closed under addition and multiplication, and a map $\psi: B \rightarrow R$. For $c \in A$ the irnage of $k\left(u_{1}+u_{2}+c u_{3}\right)$ is $R\left(v_{1}+v_{2}+\varphi(c) v_{3}\right)$. If $c \notin B$, then $k\left(u_{2}+c u_{3}\right)$ has image $R\left(p v_{2}+v_{3}\right)$ where $p \in \mathfrak{m}$ and consequently the image of $k\left(u_{1}+u_{2}+c u_{3}\right)$ must be contained on the line $R v_{1}+R\left(p v_{2}+v_{3}\right)$, which is a contradiction. Hence $A=B$ and $\varphi=\psi$. Again consider $a \notin A$. If $a^{-1} \notin A$, then $k\left(u_{1}+a u_{2}\right)=k\left(a^{-1} u_{1}+u_{2}\right)$ forces $R v_{2} \equiv R v_{1} \bmod \mathrm{m}$. Thus necessarily $a^{-1} \in A$; in particular $-1=(-1)^{-1} \in A$. Hence $A$ is a valuation ring and $\varphi: A \rightarrow R$ is a ring homomorphism.

Finally, define $N=A u_{1}+\cdots+A u_{n}$, a free $A$-module of rank $n$. Define $\beta: N \rightarrow$ $M$ by $\beta\left(\sum a_{i} u_{i}\right)=\sum \varphi\left(a_{i}\right) v_{i}$. Then $\beta$ is a $\varphi$-semilinear map. Let $x=\sum c_{i} u_{i} \in V$ with $c_{i} \in k$ not all zero. Since $A$ is a valuation ring, there is a $j$ with $c_{j}^{-1} c_{i} \in A$ for 
all $i$. By standard arguments

$$
\pi(k x)=R\left(\sum \varphi\left(c_{j}^{-1} c_{i}\right) v_{i}\right)=R \beta(k x \cap N)
$$

completing the proof.

REMARK. Clearly $N$ is not unique since it depends on the choice of the projective frames at the beginning of the proof. However, if $k$ and $R$ are commutative, a standard argument shows that the generalized place $\varphi$ and its valuation $\operatorname{ring} A$ are unique; in general they are only determined up to conjugacy. Also, $N$ and $\beta$ are determined up to multiplication by a nonzero element of $k$ and a unit of $R$, respectively. Note also that if $\varphi(a)=\infty$ then $\varphi\left(a^{-1}\right) \in \mathfrak{m}(R)$.

3. Involutions. Many of the results in this section are extensions of the work of Dieudonné $[6,7]$. Let $R$ be a local ring with 2 a unit, but not necessarily commutative, and $M$ a free $R$-module of finite rank $m \geq 3$. Let $\sigma$ be an involution in the general linear group $G L(M)=G L(m, R)$ and $P(\sigma)=\{x \in M \mid \sigma(x)=x\}$ and $N(\sigma)=\{x \in M \mid \sigma(x)=-x\}$ the positive and negative spaces of $\sigma$. Then $P(\sigma) \cap N(\sigma)=0$ and $M=P(\sigma)+N(\sigma)$ is a direct sum so that both $P(\sigma)$ and $N(\sigma)$ are free modules. The involution $\sigma$ is called extremal, or a 1 -involution, if either $P(\sigma)$ or $N(\sigma)$ has rank one. In general, $\sigma$ is an $l$-involution if either $P(\sigma)$ or $N(\sigma)$ has rank $l\left(\leq \frac{1}{2} m\right)$. Any set of mutually commuting involutions in $G L(M)$ contains at most $2^{m}$ elements and such a set can always be extended to a set of $2^{m}$ mutually commuting involutions in $G L(M)$ (see McDonald [13]). When $R$ is commutative, a set of mutually commuting involutions in the special linear group $S L(M)$ can always be extended to a maximal set of $2^{m-1}$ involutions. All of the above holds for involutions in the unitary group $U(f)$ of an anisotropic hermitian form $f$, although now we also have $V=P(\sigma) \perp N(\sigma)$ is an orthogonal sum.

PROPOSITION 1. Let $f$ be an anisotropic hermitian form which represents only one norm class. Assume $n=\operatorname{dim} V$ is odd. Let $\alpha: S U(f) \rightarrow S L(m, R)$ be a nontrivial representation where $n \geq m \geq 3$ and $R$ is a commutative local ring with 2 a unit. Then $n=m$ and $\alpha$ preserves l-involutions for $1 \leq l<\frac{1}{2} n$.

ProOF. Observe first, since $n$ is odd, that $-1_{V} \notin S U(f)$ and all extremal involutions in $S U(f)$ are of the form $-\Psi(x)$. Then $\alpha(-\Psi(x)) \neq 1_{M}$, for otherwise $\alpha$ is trivial. In fact, since any involution $\sigma$ in $S U(f)$ is a product of mutually commuting extremal involutions, it is easily seen that $\alpha(\sigma) \neq 1_{M}$ provided $\sigma \neq 1_{M}$. Any involution in $S U(f)$ can be embedded into a maximal set $S$ of $2^{n-1}$ mutually commuting involutions. Since card $\alpha(S)=\operatorname{card} S=2^{n-1}$, the image $\alpha(S)$ forms a set of $2^{n-1}$ mutually commuting involutions in $S L(m, R)$. Since $n \geq m$, this set is maximal and $n=m$. If $m=3$ then $\alpha$ necessarily preserves extremal involutions. Hence we may assume $n \geq 5$. In $S$ there are exactly $n$ extremal involutions all conjugate under $S U(f)$. Any $l$-involution in $S$, where $1<l<\frac{1}{2} n$, has at least $\frac{1}{2} n(n-1)>n$ conjugates in $S$. Since $\alpha: S \rightarrow S L(n, R)$ is injective and preserves conjugates, it follows that $\alpha$ preserves extremal involutions. Similarly, $\alpha$ preserves l-involutions.

REMARK. A slight modification of the above argument shows that a homomorphism $\alpha: U(f) \rightarrow G L(m, R)$ preserves extremal involutions for any $n \geq m \geq 3$ provided we assume card $\alpha I(f)>2$; also, it is now not necessary to assume $k$ and $R$ are commutative (see James [9, Proposition 2.7]). 
We next consider the case $n$ even. Then $-1_{V} \in S U(f)$, but $S U(f)$ contains no extremal involutions.

PROPOSITION 2. Let $f$ be an anisotropic hermitian form which represents only one norm class. Assume $n=\operatorname{dim} V \geq 6$ is even. Let $\alpha: S U(f) \rightarrow S L(m, R)$ be a nontrivial representation where $n \geq m \geq 3$ and $R$ is a commutative local ring with 2 a unit. Then $n=m$ and $\alpha$ preserves 2 -involutions.

ProOF. Any 2-involution in $S U(f)$ is of the form $\pm \Psi(x) \Psi(y)$ where $f(x, y)=0$ and can be embedded into a maximal set $S$ of $2^{n-1}$ mutually commuting involutions of $S U(f)$. Since $\alpha S U(f) \neq 1_{M}$ no noncentral involution can be killed by $\alpha$. Hence the kernel of $\alpha: S \rightarrow S L(m, R)$ is contained in $\left\{ \pm 1_{V}\right\}$. If $\alpha\left(-1_{V}\right)=-1_{M}$ then $\alpha(S)$ is a set of $2^{n-1}$ mutually commuting involutions in $S L(m, R)$ and hence $n=m$. If $\alpha\left(-1_{V}\right)=1_{M}$ and $m$ is even then $\alpha(S)$ and $-1_{M}$ generate a set of $2^{n-1}$ mutually commuting involutions in $S L(m, R)$ and again $n=m$. If, however, $m$ is odd we can only conclude $m \geq n-1$ since now $-1_{M} \notin S L(m, R)$. Assume, if possible, $n=m+1 \geq 6$. Now any noncentral element of $S$ has at least $\frac{1}{2} n(n-1)$ conjugates in $S$ and hence its image in $\alpha(S)$ has at least $\frac{1}{4} n(n-1)>n$ conjugates in $\alpha(S)$. Hence no involution in $\alpha(S)$ is extremal. Thus $\alpha(S)$ is not a maximal set of mutually commuting involutions in $S L(m, R)$ and, consequently, $m \geq n$. In all situations we now have $m=n$.

If $n=6$, then all noncentral involutions in $S L(6, R)$ are 2-involutions and hence $\alpha$ preserves 2-involutions. Assume, therefore, $n=m \geq 8$. Then any 2-involution in $S$ has exactly $\frac{1}{2} n(n-1)$ conjugates in $S$ while any other noncentral involution in $S$ has at least $\left(\begin{array}{c}n \\ 4\end{array}\right)>n(n-1)$ conjugates in $S$. Since the kernel of $\alpha: S \rightarrow S L(n, R)$ has at most 2 elements, it again follows by counting conjugates that $\alpha$ preserves 2 -involutions.

REMARK. Proposition 2 is false if $n=4$ since there exist nontrivial representations of the type $\alpha: S O_{4}(f) \rightarrow S L(3, k)$ (see Artin [1, Theorem 5.23]). A slight modification of the above proof also shows that $\alpha$ preserves 4 -involutions when $n \geq 8$.

The fact that $\alpha: S U(f) \rightarrow S L(n, R)$ preserves extremal involutions when $n$ is odd sets up a correspondence between points in $\mathbf{P} V$ and points in $\mathbf{P} M$ and hence defines a map $\pi: \mathbf{P} V \rightarrow \mathbf{P} M$. However, when $n$ is even the correspondence obtained from $\alpha$ is only between lines and we must get down to points by intersecting lines.

Assume $n \geq 8$ is even and let $\sigma, \tau$ be two commuting 2-involutions in $S L(n, R)$. Then $(\sigma, \tau)$ is called a minimal pair if $\sigma \tau$ is also a 2 -involution. The same definition applies for involutions in $U(f)$. Denote by $L(\sigma)$ the rank two subspace of $M$ (either the positive or negative space) associated with the 2-involution $\sigma$. Then $L(\sigma)$ can be viewed as a line in $\mathbf{P} M$. If $(\sigma, \tau)$ is a minimal pair then $P(\sigma, \tau)=$ $L(\sigma) \cap L(\tau)$ is a rank one direct summand of $M$ and hence a point in PM. Note, if $\sigma$ and $\tau$ are commuting 2-involutions, then $\sigma \tau$ is either $\pm 1_{M}$, a 2-involution or a 4-involution, according as the rank of $L(\sigma) \cap L(\tau)$ is 2,1 or 0 . When $n=6$, the 2-involutions and 4-involutions cannot be distinguished; hence the assumption $n \geq 8$. A homomorphism $\alpha: S U(f) \rightarrow S L(n, R)$ with nontrivial image will now map a minimal pair $(\sigma, \tau)$ in $S U(f)$ to a minimal pair $(\alpha \sigma, \alpha \tau)$ in $S L(n, R)$. We can then set up a map $\pi: \mathbf{P} V \rightarrow \mathbf{P} M$ by putting $\pi P(\sigma, \tau)=P(\alpha \sigma, \alpha \tau)$. That $\pi$ is well defined is an immediate consequence of the following result. 
Proposition 3. Take the same assumptions as Proposition 2 except $n \geq 8$ and even. Let $\sigma_{1}, \sigma_{2}$ and $\sigma_{3}$ be three 2-involutions in $S U(f)$ whose associated lines in $\mathbf{P} V$ have a unique common point, that is, $L\left(\sigma_{1}\right) \cap L\left(\sigma_{2}\right) \cap L\left(\sigma_{3}\right)=k x$. Then the lines $L\left(\alpha \sigma_{1}\right), L\left(\alpha \sigma_{2}\right)$ and $L\left(\alpha \sigma_{3}\right)$ have a common point, which is unique if $\left(\sigma_{1}, \sigma_{2}\right)$ is a minimal pair.

ProOF. The subspace $U=L\left(\sigma_{1}\right)+L\left(\sigma_{2}\right)+L\left(\sigma_{3}\right)$ of $V$ has dimension at most four. Hence there exist nonzero $y_{1}, y_{2}$ in $V$, orthogonal to $U$, with $f\left(y_{1}, y_{2}\right)=0$. Then $\tau_{1}=\Psi(x) \Psi\left(y_{1}\right), \tau_{2}=\Psi(x) \Psi\left(y_{2}\right)$ and $\tau_{3}=\tau_{1} \tau_{2}$ are mutually commuting 2-involutions in $S U(f)$. Any pair of $\tau_{1}, \tau_{2}, \tau_{3}$ form a minimal pair, in fact, $P\left(\tau_{1}, \tau_{2}\right)=k x, P\left(\tau_{1}, \tau_{3}\right)=k y_{1}$ and $P\left(\tau_{2}, \tau_{3}\right)=k y_{2}$. This constructs three points $P\left(\alpha \tau_{1}, \alpha \tau_{2}\right), P\left(\alpha \tau_{2}, \alpha \tau_{3}\right)$ and $P\left(\alpha \tau_{1}, \alpha \tau_{3}\right)$ in $\mathbf{P} M$; these three points must be distinct for otherwise $\alpha\left(\tau_{1} \tau_{2} \tau_{3}\right)=\alpha\left(1_{V}\right)$ is a 4 -involution, which is absurd. By construction $\left(\sigma_{i}, \tau_{j}\right), 1 \leq i \leq 3,1 \leq j \leq 2$, are minimal pairs; hence each $P\left(\alpha \sigma_{i}, \alpha \tau_{j}\right)$ is a point in $\mathbf{P} M$. Therefore, the line $L\left(\alpha \sigma_{i}\right)$ either contains the point $P\left(\alpha \tau_{1}, \alpha \tau_{2}\right)=L\left(\alpha \tau_{1}\right) \cap L\left(\alpha \tau_{2}\right)$, or lies in the plane (rank three direct summand) $L\left(\alpha \tau_{1}\right)+L\left(\alpha \tau_{2}\right)$ and hence $L\left(\alpha \sigma_{i}\right) \cap L\left(\alpha \tau_{3}\right) \neq 0$. But $\sigma_{i} \tau_{3}$ is a 4 -involution. Hence $\alpha\left(\sigma_{i} \tau_{3}\right)$ is also a 4 -involution and $L\left(\alpha \sigma_{i}\right) \cap L\left(\alpha \tau_{3}\right)=0$. Thus necessarily the line $L\left(\alpha \sigma_{i}\right)$ goes through the point $P\left(\alpha \tau_{1}, \alpha \tau_{2}\right)$. This point need not be the unique point of intersection since the involutions $\alpha \sigma_{i}$ can all coincide. However, the additional assumption that $\left(\sigma_{1}, \sigma_{2}\right)$ is a minimal pair ensures that the point is unique.

4. Thick collineations. Let $f$ be an anisotropic hermitian form which represents only one norm class and $\alpha: S U(f) \rightarrow S L(n, R)$ a nontrivial homomorphism where $R$ is a local ring with 2 a unit and $\operatorname{dim} f=n \geq 3$. We now use the results from the previous selection to construct a thick collineation $\pi: \mathbf{P} V \rightarrow \mathbf{P} M$ from which, with the help of the Fundamental Theorem, the main results will be obtained.

Consider first the case $n$ odd. Then for each nonzero $x$ in $V$ we have $\alpha(-\Psi(x))=$ $E(X, y)$ where $E(X, y)$ is an extremal involution in $S L(n, R)$ with negative space $X$ and positive space $R y$. Define the map $\pi: \mathbf{P} V \rightarrow \mathbf{P} M$ by $\pi(k x)=R y$.

PROPOSITION 4. Let $\alpha: S U(f) \rightarrow S L(n, R)$ be a nontrivial homomorphism with $n \geq 3$ odd. Then the map $\pi: \mathbf{P} V \rightarrow \mathbf{P} M$ induced by $\alpha$ is a collineation.

PROOF. Let $u_{1}, \ldots, u_{n}$ be any orthogonal basis of $V$ and put $\alpha\left(-\Psi\left(u_{i}\right)\right)=$ $E\left(U_{i}, v_{i}\right), 1 \leq i \leq n$. Since these involutions are mutually commutative it follows that $M=R v_{1}+\cdots+R v_{n}$ and each $U_{i}=\sum_{j \neq i} R v_{j}$ (see McDonald [13]). Hence the map $\pi$ sends the projective frame $k u_{1}, \ldots, k u_{n}$ of $\mathbf{P} V$ to the projective frame $R v_{1}, \ldots, R v_{n}$ of $\mathbf{P} M$ and consequently satisfies the first condition for a collineation.

Next let $P_{1}$ and $P_{2}$ be two points in $\mathbf{P} V$ whose images $\pi P_{1}$ and $\pi P_{2}$ span a line in $\mathbf{P} M$. We must prove that any point $P$ on the line $P_{1}+P_{2}$ has image $\pi P$ on the line $\pi P_{1}+\pi P_{2}$. Assume first that $f\left(P_{1}, P_{2}\right)=0$. Let $P_{1}=k u_{1}, P_{2}=k u_{2}$ and expand $u_{1}, u_{2}$ to an orthogonal basis $u_{1}, u_{2}, \ldots, u_{n}$ of $V$. Put $\alpha\left(-\Psi\left(u_{i}\right)\right)=E\left(U_{i}, v_{i}\right)=E_{i}$, $1 \leq i \leq n$, as above. Let $P=k x$ and $\alpha(-\Psi(x))=E(X, y)$ so that $\pi P=R y$. We must prove $y \in R v_{1}+R v_{2}$. For each $i \geq 3$ the commutator $\left[\Psi(x), \Psi\left(u_{i}{ }^{\dagger}\right]=1_{V}\right.$ and hence $E_{i} E(X, y)=E(X, y) E_{i}$. It follows that $E(X, y)\left(v_{i}\right)$ lies in the positive space $R v_{i}$ of $E_{i}$. If $E(X, y)\left(v_{i}\right)=v_{i}$ then $R y=R v_{i}$ and the positive space of the commuting involutions $E_{i}$ and $E(X, y)$ coincide, which forces the contradiction 
$\alpha\left(\Psi(x) \Psi\left(v_{i}\right)\right)=1_{M}$. Hence, necessarily $v_{i}$ lies in $X$, the negative space of $E(X, y)$, for $3 \leq i \leq n$. Let $y=\sum a_{i} v_{i}$ where $a_{i} \in R$. Then $E(X, y) E_{i}(y)=E_{i} E(X, y)(y)=$ $E_{i}(y)$ and hence $E_{i}(y) \in R y, 3 \leq i \leq n$. Thus $c y=E_{i}(y)=2 a_{i} v_{i}-y$ for either $c=1$ or $c=-1$. The first possibility again forces the contradiction $R y=R v_{i}$. Hence $c=-1$ and $a_{i}=0,3 \leq i \leq n$. Thus $y \in R v_{1}+R v_{2}$ and $\pi P$ lies on $\pi P_{1}+\pi P_{2}$ as required.

Finally assume $f\left(P_{1}, P_{2}\right) \neq 0$. Then $P_{1}+P_{2}=k u_{1}^{\prime} \perp k u_{2}^{\prime}$ and the points $P_{1}^{\prime}=k u_{1}^{\prime}$ and $P_{2}^{\prime}=k u_{2}^{\prime}$ can be expanded to an orthogonal projective frame for $\mathbf{P} V$. As above $\pi P_{1}^{\prime}+\pi P_{2}^{\prime}$ is a line in $\mathbf{P} M$ and $\pi P_{1}$ and $\pi P_{2}$ must lie on this line. Hence $\pi P_{1}+\pi P_{2}=\pi P_{1}^{\prime}+\pi P_{2}^{\prime}$ since both are rank two direct summands of $M$. Consequently, the collinearity property also holds when $f\left(P_{1}, P_{2}\right) \neq 0$.

We next consider the case $n \geq 8$ even. Then, as explained in the previous section, a nontrivial homomorphism $\alpha: S U(f) \rightarrow S L(n, R)$ induces a map $\pi: \mathbf{P} V \rightarrow \mathbf{P} M$ via its action on minimal pairs of 2 -involutions in $S U(f)$.

Proposition 5. Let $\alpha: S U(f) \rightarrow S L(n, R)$ be a nontrivial homomorphism with $n \geq 8$ even. Then the map $\pi: \mathbf{P V} \rightarrow \mathbf{P} M$ induced by $\alpha$ is a collineation.

ProOF. Let $u_{1}, \ldots, u_{n}$ be an orthogonal basis of $V$ and put $\sigma_{i}=\Psi\left(u_{i}\right) \Psi\left(u_{i+1}\right)$, $1 \leq i \leq n$, where we view the subscipts $i$ modulo $n$. Then each $\left(\sigma_{i-1}, \sigma_{i}\right)$ is a minimal pair in $S U(f)$ with associated point $P\left(\sigma_{i-1}, \sigma_{i}\right)=k u_{i}$. Let $L\left(\sigma_{i}\right)$ denote the 2-dimensional space of $\sigma_{i}$ (now the negative space). Each $L\left(\sigma_{i}\right)=k u_{i} \perp k u_{i+1}$ can be viewed as a line in $\mathbf{P} V$. The image of the point $k u_{i}$ under the map $\pi$ is the point $P\left(\alpha \sigma_{i-1}, \alpha \sigma_{i}\right)=R v_{i}$, say, of $\mathbf{P} M$. Then, from the definition of $\pi$, we have $R v_{i}=L\left(\alpha \sigma_{i-1}\right) \cap L\left(\alpha \sigma_{i}\right)$. Hence $R v_{i}+R v_{i+1} \subseteq L\left(\alpha \sigma_{i}\right)$. Whenever $|i-j| \geq 2$ the product $\sigma_{i} \sigma_{j}$ is a 4 -involution and since $\alpha$ preserves 4 -involutions, it follows that $L\left(\alpha \sigma_{i}\right) \cap L\left(\alpha \sigma_{j}\right)=0$. Hence $R v_{i} \cap R v_{i+1}=0$ and, consequently, $L\left(\alpha \sigma_{i}\right)=R v_{i}+R v_{i+1}$. Since each $L\left(\alpha \sigma_{i}\right)$ is a direct summand of $M$, we now have $M=R v_{1}+\cdots+R v_{n}$ and $\pi$ carries the projective frame $k u_{1}, \ldots, k u_{n}$ of $\mathbf{P} V$ to the projective frame $R v_{1}, \ldots, R v_{n}$ of $\mathbf{P} M$.

If $P_{1}$ and $P_{2}$ are two orthogonal points in $\mathrm{P} V$ whose images $\pi P_{1}$ and $\pi P_{2}$ generate a line $\pi P_{1}+\pi P_{2}$ then we may take $P_{1}=k u_{1}$ and $P_{2}=k u_{2}$ as above. Let $P$ be any point on the line $P_{1}+P_{2}$ and choose a minimal pair $\left(\sigma_{1}, \tau\right)$ of 2 -involutions, with $\sigma_{1}$ as before, and $P=P\left(\sigma_{1}, \tau\right)$. Then $\pi P$ lies on $L\left(\alpha \sigma_{1}\right)=\pi P_{1}+\pi P_{2}$ as required. The case $f\left(P_{1}, P_{2}\right) \neq 0$ can be handled as in Proposition 4 .

\section{Proposition 6. The collineations of Propositions 4 and 5 are thick.}

Proof. Let $P_{1}=k u_{1}$ and $P_{2}=k u_{2}$ be points in $\mathrm{P} V$ with images $\pi P_{1}=R v_{1}$ and $\pi P_{2}=R v_{2}$ spanning a line in $\mathbf{P} M$. We may assume $f\left(u_{1}, u_{1}\right)=f\left(u_{2}, u_{2}\right)$. Consider first $f\left(u_{1}, u_{2}\right)=0$ and expand $u_{1}, u_{2}$ to an orthogonal basis $u_{1}, \ldots, u_{n}$ of $V$. By symmetry $R\left(v_{1}+p v_{2}\right)$ is the image of $k\left(u_{1}+u_{2}\right)$. If $p$ is a unit then $\pi$ is thick. Assume, therefore, $p \in \mathfrak{m}$. Let $k\left(u_{1}-u_{2}\right)$ have image $R\left(a_{1} v_{1}+a_{2} v_{2}\right)$. Since $u_{1}+u_{2}, u_{1}-u_{2}, u_{3}, \ldots, u_{n}$ is an orthogonal basis of $V$ it follows, as before, that $R\left(v_{1}+p v_{2}\right)$ and $R\left(a_{1} v_{1}+a_{2} v_{2}\right)$ must span $R v_{1}+R v_{2}$. Hence $a_{2}$ is a unit which can be made 1. Again we are finished unless $a_{1}=q \in \mathfrak{m}$. If $n$ is odd, apply $\alpha$ to the identity $\Psi\left(u_{2}\right) \Psi\left(u_{1}+u_{2}\right)=\Psi\left(u_{1}-u_{2}\right) \Psi\left(u_{2}\right)$. Then, as in Proposition 4, 
$E\left(U_{2}, v_{2}\right) E\left(X, v_{1}+p v_{2}\right)=E\left(Y, q v_{1}+v_{2}\right) E\left(U_{2}, v_{2}\right)$ where $U_{2}=\sum_{i \neq 2} R v_{i}$. Hence

$$
\begin{aligned}
E\left(Y, q v_{1}+v_{2}\right)\left(-v_{1}+p v_{2}\right) & =E\left(Y, q v_{1}+v_{2}\right) E\left(U_{2}, v_{2}\right)\left(v_{1}+p v_{2}\right) \\
& =E\left(U_{2}, v_{2}\right) E\left(X, v_{1}+p v_{2}\right)\left(v_{1}+p v_{2}\right) \\
& =E\left(U_{2}, v_{2}\right)\left(v_{1}+p v_{2}\right)=-v_{1}+p v_{2}
\end{aligned}
$$

and, therefore, $-v_{1}+p v_{2} \in R\left(q v_{1}+v_{2}\right)$. This is impossible since $q \in \mathfrak{m}$. When $n$ is even a similar calculation can be made starting with the 2-involution identity $\sigma_{2} \Psi\left(u_{3}\right) \Psi\left(u_{1}+u_{2}\right)=\Psi\left(u_{1}-u_{2}\right) \Psi\left(u_{3}\right) \sigma_{2}$, where $\sigma_{2}=\Psi\left(u_{2}\right) \Psi\left(u_{3}\right)$. The remaining case with $f\left(u_{1}, u_{2}\right) \neq 0$ is handled as in Proposition 4.

5. Representations over local rings. Let $G$ be a subgroup of $U(f)$ containing $S U(f)$ and $\alpha: G \rightarrow G L(M)$ a representation, with $\alpha S U(f)$ nontrivial, where $M$ is a free module over a commutative local ring $R$ with 2 a unit and $n=\operatorname{dim} f=$ rank $M \geq 3$. Since $S U(f)$ is perfect, $\alpha S U(f) \subseteq S L(n, R)$. Then except for $n=4$ or 6 the homomorphism $\alpha$ induces a thick collineation $\pi: \mathbf{P} V \rightarrow \mathbf{P} M$. The cases $n=4$ and 6 can be included if the hypotheses are modified to $I(f) \subseteq G$ and card $\alpha I(f)>2$. By the Fundamental Theorem there now exists a generalized place $\varphi: k \rightarrow R \cup \infty$ with valuation ring $A=\varphi^{-1}(R)$ and homomorphism $\varphi: A \rightarrow R$, a free $A$-module $N$ in $V$ of $\operatorname{rank} n$ and a $\varphi$-semilinear map $\beta: N \rightarrow M$ inducing $\pi$. Thus $\pi P=R \beta(P \cap N)$ for any point $P$ in $\mathbf{P} V$. We study these objects further and use them to describe the original representation $\alpha$ via a twisted congruence subgroup in $G$.

We first establish that $A^{*}=A$ and $\mathfrak{m}(A)^{*}=\mathfrak{m}(A)$. The $A$-module $N$ in $V$ is called maximal if $N=\{x \in V \mid f(x, x) \in \mathfrak{c}\}$ for some fractional ideal $\mathfrak{c}$ of $A$. Denote by $U(N)$ the subgroup of $U(f)$ consisting of all $\sigma \in U(f)$ such that $\sigma(N)=N$. If $N$ is maximal then $U(N)=U(f)$.

Proposition 7. (i) $A^{*}=A$ and $\mathfrak{m}(A)^{*}=\mathfrak{m}(A)$.

(ii) The A-module $N$ is maximal and has an orthogonal basis.

(iii) $U(N)=U(f)$.

ProOF. Let $x, y \in N$ be primitive (that is, $x, y$ are not in $\mathfrak{m}(A) N)$ with $\pi(k x) \neq$ $\pi(k y)$. Assume, if possible, $c=2 f(x, x)^{-1} f(x, y)$ is not in $A$, or equivalently, that $f(x, y)^{-1} f(x, x) \in \mathfrak{m}(A)$. Let $\beta(x)=v$ and $\beta(y)=w$ so that $R v \neq R w$. Then

$$
\Psi(x) \Psi(y)=\Psi(\Psi(x)(y)) \Psi(x)=\Psi(y-c x) \Psi(x)
$$

is an identity in $S U(f)$. If $n$ is odd, applying the homomorphism $\alpha$ gives an identity $E(X, v) E(Y, w)=E\left(Z, \varphi\left(c^{-1}\right) w-v\right) E(X, v)$ in $S L(M)$. Hence $E(X, v)(w) \in$ $R\left(\varphi\left(c^{-1}\right) w-v\right)$. But $E(X, v)(w)=-w+2 a v$ for some $a \in R$ which forces the contradiction $R v=R w$. If $n \geq 8$ is even, choose $z$ primitive in $N$ and orthogonal to $x$ and $y$. Then a similar contradiction can be achieved using the 2 -involutions $\Psi(x) \Psi(z), \Psi(y) \Psi(z)$ and $\Psi(y-c x) \Psi(z)$. Hence, in both cases, $f(x, y) \in f(x, x) A$.

Since $A$ is a valuation ring the module $N$ has an orthogonal decomposition into indecomposable components of rank one or two. If $N$ has an indecomposable binary component $B=A x+A y$, then necessarily $f(x, x) \in f(x, y) \mathfrak{m}(A)$. This gives a contradiction with the previous paragraph since $\beta(x), \beta(y)$ expands to a basis of $M$. Thus $N$ has an orthogonal basis and we may assume $N=A u_{1} \perp \cdots \perp A u_{n}$ and $M=R v_{1}+\cdots+R v_{n}$ where $\beta\left(u_{i}\right)=v_{i}, 1 \leq \imath \leq n$. Let $f\left(u_{i}, u_{i}\right)=a_{i}$, 
$1 \leq i \leq n$, where each $a_{i}$ is a nonzero element of $k$. Since $A$ is a valuation ring there exists $a_{1}$, say, such that $a_{1}^{-1} a_{i} \in A, 1 \leq i \leq n$.

Let $e \in A$ be a unit. If $e^{*} \notin A$ replace $e$ by $e^{-1}$. Hence $e^{*} \in A$. Assume $e^{*}$ is not a unit, so $e^{*} \in \mathfrak{m}(A)$. Put $x=e^{*} u_{1}+e u_{2}$ and $y=u_{1}$. Then $\pi(k x) \neq \pi(k y)$ and consequently $f(x, y) \in f(x, x) A$. Therefore, $e a_{1} \in\left(e a_{1} e^{*}+e^{*} a_{2} e\right) A$ which gives the contradiction $1 \in \mathfrak{m}(A)$. Thus $e^{*}$ must be a unit. Hence $(1+\mathfrak{m}(A))^{*} \subseteq A$, so that $\mathfrak{m}(A)^{*} \subseteq A$. It follows that $A^{*}=A$ and $\mathfrak{m}(A)^{*}=\mathfrak{m}(A)$.

Next let $p \in \mathfrak{m}(A)$ and put $x=p^{*} u_{1}+u_{i}, i \neq 1$, and $y=u_{1}$. Then $\pi(k x) \neq \pi(k y)$ so that $f(x, y) \in f(x, x) A$. Therefore, $p a_{1}$ lies in the fractional ideal $\left(p a_{1} p^{*}+a_{i}\right) A$ and, since $A$ is a valuation ring, $p a_{1} \in a_{i} A$. Thus $a_{1} \mathfrak{m}(A) \subseteq a_{i} A$. If $\mathfrak{m}(A)$ is not a principal ideal then $a_{1}^{-1} a_{i}$ must be a unit, since $a_{1}^{-1} a_{i} \in A$. In this case $N$ is now a modular lattice. If, on the other hand, $\mathfrak{m}(A)$ is a principal ideal then either $a_{1}^{-1} a_{i}$ is a unit, or $a_{i} A=a_{1} \mathfrak{m}(A)$. The second possibility cannot occur since $f$ represents only one norm class. Thus again $N$ is modular.

Finally, let $x=\sum c_{i} u_{i} \in N$ with at least one coefficient, say $c_{1}$, a unit in $A$. Assume $f(x, x) \in a_{1} \mathfrak{m}(A)$, so that necessarily a second coefficient, say $c_{2}$, is also a unit. Put $y=u_{2}$. Then $\pi(k x) \neq \pi(k y)$ and $f(x, y) \in f(x, x) A$. This forces the contradiction $1 \in \mathfrak{m}(A)$. Hence $f(x, x) A=a_{1} A$ and $N$ is now necessarily a maximal lattice.

DEFINITION. Let $\chi: G \rightarrow \mathfrak{u}(R)$ be a character, that is, a homomorphism from $G$ into the group of units $\mathfrak{u}(R)$ of $R$, and $\mathfrak{a}$ the kernel of $\varphi: A \rightarrow R$. Then $G(\mathfrak{a}, \chi)$ denotes the twisted congruence subgroup of the group $G$ consisting of all $\sigma \in G$ such that

(i) $\chi(\sigma) \in \varphi(A)$, so $\chi(\sigma)=\varphi\left(a_{\sigma}\right)$ for some $a_{\sigma} \in A$,

(ii) $a_{\sigma} \sigma(x) \equiv x \bmod \mathfrak{a} N$ for all $x \in N$.

Note that the definition is independent of the choice of $a_{\sigma}$ since $\mathfrak{a}$ is the kernel of $\varphi$ and condition (ii) is modulo $\mathfrak{a} N$.

PROOF OF THEOREM 1. Many parts of the theorem have already been established. It remains to construct a character $\chi: G \rightarrow \mathfrak{u}(R)$, show that $U(\mathfrak{a}, \chi)$ is the kernel of $\alpha$, and describe the action of $\alpha \sigma$ for $\sigma \in G$.

Consider first $n \geq 3$ odd. Let $x \in N$ be primitive and $N=A x \perp X$. Then the image of $-\Psi(x) \in S U(f)$ under $\alpha$ is $E(Y, \beta(x))$ where $Y=\beta(X)$. For if $w \in X$ is primitive, then $\Psi(x)$ and $\Psi(w)$ commute and hence $E(Y, \beta(x))$ and $E(Z, \beta(w))$ commute. Since $R \beta(x) \cap R \beta(w)=0$, for otherwise $\alpha(\Psi(x) \Psi(w))=$ $1_{M}$, it follows that $\beta(w) \in Y$. Hence $\beta(X) \subseteq Y$ and, since they have the same rank, $\beta(X)=Y$. For any $\sigma \in G$ and primitive $x \in N$ we have $\sigma(-\Psi(x)) \sigma^{-1}=$ $-\Psi(\sigma(x))$ in $G$. Applying the homomorphism $\alpha$ gives $(\alpha \sigma) E(\beta(X), \beta(x))(\alpha \sigma)^{-1}=$ $E(\beta \sigma(X), \beta \sigma(x))$. Hence, from comparing the positive spaces,

$$
(\alpha \sigma)(\beta(x))=\chi(\sigma, x) \beta \sigma(x)
$$

for some unit $\chi(\sigma, x)$ in $R$. We show $\chi(\sigma)=\chi(\sigma, x)$ is independent of the choice of $x$. Let $y \in N$ be primitive with $f(x, y)=0$. Then $x+y \in N$ is also primitive. Moreover, if $\alpha(-\Psi(x))=E(Y, \beta(x))$, then $\beta(y) \in Y$ and hence $R \beta(x) \cap R \beta(y)=0$. Then, from $\chi(\sigma, x+y) \beta \sigma(x+y)=(\alpha \sigma) \beta(x+y)=\chi(\sigma, x) \beta \sigma(x)+\chi(\sigma, y) \beta \sigma(y)$ it follows that $\chi(\sigma, x+y)=\chi(\sigma, x)=\chi(\sigma, y)$. Thus $\chi(\sigma, x)$ is independent of $x$ and we have constructed a map $\chi: G \rightarrow \mathfrak{u}(R)$. It is easily seen that $\chi$ is a group 
homomorphism. Also, we have established that

$$
(\alpha \sigma)(\beta(x))=\chi(\sigma) \beta \sigma(x) .
$$

Now let $n \geq 8$ be even. Let $\tau_{1}=\Psi(x) \Psi(y)$ and $\tau_{2}=\Psi(x) \Psi(z)$ where $x, y, z$ are primitive orthogonal elements of $N$. Then $\left(\tau_{1}, \tau_{2}\right)$ is a minimal pair of 2 -involutions with associated point $P\left(\tau_{1}, \tau_{2}\right)=L\left(\tau_{1}\right) \cap L\left(\tau_{2}\right)=k x$. From the construction of $\pi$ in Proposition 5 and the definition of $\beta$ we have $L\left(\alpha \tau_{1}\right)=\beta L\left(\tau_{1}\right), L\left(\alpha \tau_{2}\right)=\beta L\left(\tau_{2}\right)$ and $P\left(\alpha \tau_{1}, \alpha \tau_{2}\right)=\beta P\left(\tau_{1}, \tau_{2}\right)$. For any $\sigma \in G,\left(\sigma \tau_{1} \sigma^{-1}, \sigma \tau_{2} \sigma^{-1}\right)$ remains a minimal pair as also does its image under $\alpha$. Since $L\left(\sigma \tau_{1} \sigma^{-1}\right)=\sigma L\left(\tau_{1}\right), L\left(\sigma \tau_{2} \sigma^{-1}\right)=\sigma L\left(\tau_{2}\right)$ and $P\left(\sigma \tau_{1} \sigma^{-1}, \sigma \tau_{2} \sigma^{-1}\right)=\sigma P\left(\tau_{1}, \tau_{2}\right)$ it follows that $(\alpha \sigma) \beta(x)=\chi(\sigma, x) \beta \sigma(x)$ for some unit $\chi(\sigma, x)$ in $R$. Again it follows that $\chi(\sigma, x)$ is independent of $x$ and that $\chi$ induces a group homomorphism from $G$ to the units of $R$.

If $\mathfrak{a}$ is the kernel of the homomorphism $\varphi: A \rightarrow R$ then clearly $\mathfrak{a} N$ is the kernel of the $\varphi$-semilinear map $\beta: N \rightarrow M$. Finally we must show that the twisted congruence subgroup $G(\mathfrak{a}, \chi)$ is the kernel of $\alpha$. Let $\sigma \in \operatorname{ker} \alpha$. Then $\alpha(\sigma)=1_{M}$ and consequently $\beta(x)=\chi(\sigma) \beta \sigma(x)$ for any $x \in N$. Hence $\chi(\sigma) \in \varphi(A)$. Therefore, $\beta\left(x-a_{\sigma} \sigma(x)\right)=0$ where $\varphi\left(a_{\sigma}\right)=\chi(\sigma)$. Thus $\sigma \in G(\mathfrak{a}, \chi)$. The converse is similar. This completes the proof of Theorem 1 .

REMARK. It is quite possible for $\mathfrak{a}=0$ and $\varphi$ and $\beta$ to be injections. The kernel of $\alpha$ is then contained in the center of $U(f)$.

Proposition 8. Let $\varphi: k \rightarrow R \cup \infty$ be the generalized place in Theorem 1 and $A=\varphi^{-1}(R)$ the associated valuation ring. Let $K$ be the fixed field of $k$ under the involution * ${ }^{*}$. Then

(i) $\mathbf{Q} \subseteq A$,

(ii) if $\mathbf{R} \subseteq K$ then $\mathbf{R} \subseteq A$,

(iii) $B / \mathfrak{m}(B)$ is formally real, where $B=K \cap A$ is the induced valuation ring of $K$.

PROOF. Since $\varphi(1)=1$ we know $\mathbf{Z} \subseteq A$. Assume $\varphi(p) \in \mathfrak{m}(R)$ for some odd prime $p$ in Z . Then $p \in \mathfrak{m}(A)$. Let $N=A u_{1} \perp \cdots \perp A u_{n}$ be the maximal lattice in Theorem 1 where we may assume $f\left(u_{i}, u_{i}\right)=a \neq 0,1 \leq i \leq n$. Since the quadratic form $\langle 1,1, \ldots, 1\rangle$ is isotropic over the finite field $\mathbf{F}_{p}$ there exist integers $c_{1}, \ldots, c_{n}$ with $c_{1}=1$ such that $\sum c_{i}^{2} \in p \mathbf{Z}$. Then $x=\sum c_{i} u_{i} \in N$ is primitive and $f(x, x) \in a \mathfrak{m}(A)$, since the involution is trivial on $\mathbf{Z}$. This contradicts the fact that $N$ is a maximal lattice (Proposition 7). Hence $\varphi(p)$ is a unit in $R$ for all odd primes $p$. By hypothesis 2 is a unit in $R$ and hence also in $A$. It follows that $\mathbf{Q} \subseteq A$ and the valuation induced by $\varphi$ on $\mathbf{Q}$ is trivial.

Now assume $\mathbf{R} \subseteq K$. Let $a \in \mathbf{R}$ with $0<a<1$. Assume $\varphi(a)=\infty$ so that $a^{-1} \in \mathfrak{m}(A)$. Let $a^{-1}-1=b^{2}$ where $b \in \mathbf{R}$. Then $x=b u_{1}+u_{2}$ is primitive in $N$ but $f(x, x) \in a \mathfrak{m}(A)$, contradicting the fact that $N$ is maximal. Therefore, $\mathbf{R} \subseteq A$ by the Archimedean axiom on $\mathbf{R}$, and the induced valuation on $\mathbf{R}$ is now trivial. The proof of (iii) is similar.

REMARK. Proposition 8(ii) is more general. The same result holds for any subfield of $\mathbf{R}$ all of whose positive elements are squares. If $k$ is such a field then Theorem 1 suggests that the only nontrivial normal subgroup of the orthogonal group $S O(f), n \neq 4$, is its center. This is true, see Bröcker [4]. A similar result should hold for unitary groups $S U(f)$ over $k(i), i^{2}=-1$. 
6. Representations over commutative rings. We now extend the results of the previous section to representations $\alpha: G \rightarrow G L(n, R)$, where $R$ is a commutative ring with 2 not a zero divisor. If 2 is not a unit in $R$, enlarge $R$ by localizing with respect to the multiplicative set generated by 2 . Assume, therefore, 2 is a unit in $R$. Let $\mathfrak{p}$ be a prime ideal of $R, R_{\mathfrak{p}}$ the localization at $\mathfrak{p}$ and $\varepsilon_{\mathfrak{p}}: R \rightarrow R_{\mathfrak{p}}$ the canonical homomorphism. Localize $M$ at $\mathfrak{p}$ and let $M \rightarrow M_{\mathfrak{p}}$ be the natural extension of $\varepsilon_{\mathfrak{p}}$. Then we have a homomorphism $\eta_{\mathfrak{p}}: G L(M) \rightarrow G L\left(M_{\mathfrak{p}}\right)$ from the group $G L(M)=G L(n, R)$ to the group $G L\left(M_{\mathfrak{p}}\right)=G L\left(n, R_{\mathfrak{p}}\right)$.

PROOF OF THEOREM 2. Let $\alpha: S U(f) \rightarrow G L(M)$ be a nontrivial representation of $S U(f)$. Then Theorem 1 can be applied to the composite map $\eta_{\mathfrak{p}} \circ \alpha: S U(f) \rightarrow G L\left(n, R_{\mathfrak{p}}\right)$. If the image of $S U(f)$ is now trivial then obviously the kernel is $S U(f)$. Otherwise the kernel is a twisted congruence subgroup $S U\left(\mathfrak{a}_{\mathfrak{p}}, \chi_{\mathfrak{p}}\right)$ of $S U(f)$. Let $H$ be the intersection of all these twisted congruence subgroups for which $\eta_{\mathfrak{p}} \circ \alpha$ is nontrivial as $\mathfrak{p}$ varies over the maximal ideals of $R$. Clearly $H$ is a normal subgroup of $S U(f)$ containing the kernel of $\alpha$. Conversely, let $\sigma \in H$. Then $\eta_{\mathfrak{p}}(\alpha \sigma)=1$ for all maximal ideals $\mathfrak{p}$. Hence $(\alpha \sigma(x)-x)_{\mathfrak{p}}=0$ in $M_{\mathfrak{p}}$ for all maximal ideals $\mathfrak{p}$ and all $x \in M$. It follows (for example, Bass [2, p. 108]) that $(\alpha \sigma)(x)=x$ in $M$ for all $x \in M$. Thus $\sigma \in \operatorname{ker} \alpha$ and

$$
1 \rightarrow H \rightarrow S U(f) \stackrel{\alpha}{\rightarrow} G L(n, R)
$$

is an exact sequence. Finally, since $S U(f)$ is perfect, the characters $\chi_{\mathfrak{p}}: S U(f) \rightarrow$ $\mathfrak{u}\left(R_{\mathfrak{p}}\right)$ are all trivial.

To prove Theorem 3 we need to study the generalized places $\varphi: \mathbf{C}(X) \rightarrow R \cup \infty$, with $R$ a local ring, extending an injection $\varphi: \mathbf{C} \rightarrow R$. Recall the notation $A_{c}$ and $\varphi_{c, m}$ from the introduction.

Proposition 9. Let $\varphi: \mathbf{C}(X) \rightarrow R \cup \infty$ be a generalized place, which induces the trivial valuation on $\mathbf{C}$, and $A=\varphi^{-1}(R)$ the associated valuation ring. Then one of the following occurs:

(i) $A=\mathbf{C}(X)$ and $\varphi$ is an injection into $R$,

(ii) $A=A_{c}$ and $\varphi=\varphi_{c, m}$ for some $c \in \mathbf{C}$ and integer $m \geq 1$,

(iii) $A=A_{\infty}$ and $\varphi=\varphi_{\infty, m}$ for some integer $m \geq 1$.

ProOf. We have $\varphi(\mathbf{C})=\mathbf{C} \subseteq R$. Let $\mathfrak{a}$ be the kernel of $\varphi: A \rightarrow R$. Assume first $\varphi(X)=x \in R$. Then $\mathbf{C}[x] \subseteq R$. If $g(x)$ is a unit in $R$ for all nonzero polynomials $g(X) \in \mathbf{C}[X]$ then $A=\mathbf{C}(X), \mathfrak{a}=0$ and $\varphi$ is an injection. Assume, therefore, there exists a monic nonconstant polynomial $g(X) \in \mathbf{C}[X]$ with $g(x) \in \mathfrak{m}(R)$. Let $g(X)$ have minimal degree and $c \in \mathbf{C}$ be a root of $g(X)$. Then $g(X)=X-c$. If $h(X) \in \mathbf{C}[X]$ is a polynomial relatively prime to $g(X)$, then $h(x)$ must be a unit of $R$, for otherwise $1 \in \mathfrak{m}(R)$. Hence $A=A_{c}$ and $\mathfrak{m}(A)=(X-c) A_{c}$. Since the valuation ring $A_{c}$ is discrete, the kernel $\mathfrak{a}=(X-c)^{m} A_{c}$ for some integer $m \geq 1$. Then $\varphi=\varphi_{c, m}$. Finally assume $\varphi(X)=\infty$. Then $X^{-1} \in \mathfrak{m}(A)$ and $\varphi\left(X^{-1}\right)=x \in \mathfrak{m}(R)$. Hence $\varphi\left(X^{-1}-c\right)$ is a unit of $R$ for all nonzero $c \in \mathbf{C}$. Therefore, $(X-a) /(X-b)$ is a unit in $A$ for all $a, b \in \mathbf{C}$. Consequently $A=A_{\infty}$ consists of all rational functions with degree $\leq 0$. Hence $\mathrm{m}(A)=X^{-1} A_{\infty}$ and the kernel $\mathfrak{a}=X^{-m} A_{\infty}$ for some $m \geq 1$. Thus $\varphi=\varphi_{\infty, m}$.

PROOF OF THEOREM 3. We apply Theorems 1 and 2 to the special case $k=\mathbf{C}(X)$. Let $\varphi: \mathbf{C}(X) \rightarrow R \cup \infty$ be a generalized place occurring in Theorem 
1(i). By Proposition 8, $\varphi(\mathbf{R})=\mathbf{R} \subseteq R$ (after identifying $\mathbf{R}$ with a subfield of $R$ ). Also $\varphi(i)^{2}=\varphi(-1)=-1$ and hence $\varphi(\mathbf{C})=\mathbf{C} \subseteq R$. Therefore $\varphi$ is one of the generalized places in Proposition 9. However, if $\varphi=\varphi_{c, m}$ with $c \in \mathbf{C}$, then $c \in \mathbf{R}$ since by Theorem 1 (i) we must have $A_{c}^{*}=A_{c}$. Let $H$ be a nontrivial normal subgroup of $S U(f)$ as in Theorem 3. By Theorem $2, H=\bigcap S U\left(\mathfrak{a}_{j}, 1\right)$ where the $\mathfrak{a}_{j}$ are kernels of generalized places $\varphi_{j}: \mathbf{C}(x) \rightarrow R_{j} \cup \infty$. We may assume $\mathfrak{a}_{j} \neq 0$ and hence any injective $\varphi_{j}$ can be ignored. Only a finite number of $\mathfrak{a}_{j}$ can be nonzero, for otherwise $H=1$ is trivial. Let $A$ be the intersection of the finite number of valuation rings $A_{c}, c \in \mathbf{R} \cup \infty$, associated with generalized places with nontrivial kernel, and $\mathfrak{a}$ the intersection of these kernels. Then $\mathfrak{a}$ is an ideal of $A$. By the weak approximation theorem for valuations we can choose a common basis $u_{1}, \ldots, u_{n}$ for all the associated maximal lattices in Theorem 1(ii). Then $N=A u_{1}+\cdots+A u_{n}$ is the intersection of these maximal lattices and hence $\sigma(N)=N$ for all $\sigma \in S U(f)$. Let $S U(\mathfrak{a})$ be the congruence subgroup of all $\sigma \in S U(f)$ with $(\sigma-1) N \subseteq \mathfrak{a} N$. Then $H=\bigcap S U\left(\mathfrak{a}_{j}, 1\right)=S U(\mathfrak{a})$, completing the proof.

\section{REFERENCES}

1. E. Artin, Geometric algebra, Interscience, New York, 1957.

2. H. Bass, Algebraic K-theory, Benjamin, New York, 1968.

3. A. Borel and J. Tits, Homomorphismes "abstraits" de groupes algébriques simples, Ann. of Math. 97 (1973), 499-571.

4. L. Bröcker, Zur orthogonalen Geometrie über pythagoreischen Körpern, J. Reine Angew. Math. 268/269 (1974), 68-77.

5. C.-N. Chang, Integral orthogonal groups over $\mathbf{R}\left(\left(\pi_{1}\right)\right)\left(\left(\pi_{2}\right)\right)-\left(\left(\pi_{n}\right)\right)$, J. Algebra 39 (1976), 308327.

6. J. Dieudonné, On the automorphisms of the classical groups, Mem. Amer. Math. Soc. No. 2, 1951, 95 pp.

7. __ La ǵeometrié des groupes classiques, Springer, Berlin-Heidelberg-New York, 1971.

8. A. J. Hahn, D. G. James and B. Weisfeiler, Homomorphisms of algebraic and classical groups: a survey, Canad. Math. Soc. Conf. Proc., vol. 4, Amer. Math. Soc., Providence, R.I., 1984, pp. $249-296$.

9. D. G. James, Projective geometry for orthogonal groups, J. Reine Angew. Math. 319 (1980), $104-117$.

10. _ Homomorphisms of unitary groups, Math. Z. 178 (1981), 343-352.

11. D. James, W. Waterhouse and B. Weisfeiler, Abstract homomorphisms of algebraic groups: problems and bibliography, Comm. Algebra 9 (1981), 95-114.

12. T. Y. Lam, The algebraic theory of quadratic forms, Benjamin, Reading, Mass., 1973.

13. B. R. McDonald, Geometric algebra over local rings, Dekker, New York, 1976.

14. B. Pollak, Orthogonal groups over R(( $\pi)$ ), Amer. J. Math. 100 (1968), 214-230.

15. J. Tits, Homomorphismes "abstrait" de groupes de Lie, Sympos. Math., vol. 13 (Inst. Naz. Alta Mat., Rome, 1972), Academic Press, London, 1974, pp. 479-499.

16. B. Weisfeiler, On abstract homomorphisms of anisotropic algebraic groups over real-closed fields, J. Algebra 60 (1979), $485-519$.

17. _ Abstract isomorphisms of simple algebraic groups split by quadratic extensions, J. Algebra 68 (1981), 335368.

Department of Mathematics, Pennsylvania State University, University PARK, PENNSYLVANIA 16802 